1 Title

\title{
Regulation of single-cell genome organization into TADs and chromatin nanodomains
}

\section{Authors list and affiliations}

Quentin Szabo $^{1}$, Axelle Donjon ${ }^{1 *}$, Ivana Jerković ${ }^{1 *}$, Giorgio L. Papadopoulos ${ }^{1 *}$, Thierry Cheutin ${ }^{1}$, Boyan Bonev ${ }^{1,5}$, Elphege P. Nora ${ }^{2}$, Benoit B. Bruneau ${ }^{2,3,4}$, Frédéric Bantignies ${ }^{1 \#}$ \& Giacomo Cavalli ${ }^{1 \#}$ 8

${ }^{1}$ Institute of Human Genetics, CNRS, University of Montpellier, Montpellier, France

${ }^{2}$ Cardiovascular Research Institute, University of California, San Francisco, CA 94158 USA

${ }^{3}$ Gladstone Institutes, San Francisco, CA, 94158 USA

${ }^{4}$ Department of Pediatrics, University of California, San Francisco, San Francisco, CA 94143 USA

${ }^{5}$ Present Address: Helmholtz Pioneer Campus, Helmholtz Zentrum München, 85764 Neuherberg, Germany

${ }^{*}$ These authors contributed equally to this work \# Corresponding authors: frederic.bantignies@igh.cnrs.fr (F.B.) and giacomo.cavalli@igh.cnrs.fr (G.C.)

\section{Abstract}

The genome folds into a hierarchy of three-dimensional (3D) structures within the nucleus. At the sub-megabase scale, chromosomes form topologically associating domains (TADs) ${ }^{1-4}$. However, how TADs fold in single-cells remains elusive. Here, we revealed TAD features inaccessible to cell-population analysis by using super-resolution microscopy. TAD structures and physical insulation associated with their borders are variable between individual cells, yet chromatin intermingling is enriched within TADs compared to adjacent TADs in most cells. The spatial segregation of TADs is further exacerbated during cell differentiation. Favored interactions within TADs are regulated by cohesin and CTCF through distinct mechanisms: cohesin generates chromatin contacts and intermingling while CTCF prevents inter-TAD contacts. Furthermore, TADs are subdivided into discrete nanodomains which persist in cells depleted of CTCF or cohesin, whereas disruption of nucleosome contacts alters their structural 

chromosomes at the nanoscale.

\section{Introduction}

Chromosome folding is a complex process involving a hierarchy of architectural layers, from nucleosome clutches ${ }^{5,6}$ to chromosome territories ${ }^{7}$. High-throughput chromosome conformation capture (Hi-C) studies revealed that, at the sub-megabase scale, the genome is partitioned into TADs, which have been proposed to delimit regulatory landscapes where functional interactions between genes and distal cis-regulatory elements occur ${ }^{8}$. In mammals, most TADs are defined by the binding of CTCF in a convergent manner at their borders and by the action of the cohesin complex ${ }^{9-16}$. However, the structure of mammalian TADs and their association with genome activity in individual cells are still under debate. Single-cell $\mathrm{Hi}-\mathrm{C}(\mathrm{scHi}-\mathrm{C})$ studies described heterogenous chromatin contacts, suggesting that TADs reflect probabilistic preferential interactions rather than stable chromosomal domains ${ }^{17-19}$. On the other hand, microscopy analyses revealed "nanocompartments" that largely correspond to TADs in flies ${ }^{20}$, and "TAD-like" structures were recently observed in individual mammalian cells ${ }^{21}$, but their exact relationship with the TADs defined in Hi-C is unclear.

\section{Results}

To investigate single-cell chromosome architecture at the sub-megabase scale, we combined fluorescent in-situ hybridization (FISH) with Oligopaint ${ }^{22,23}$ and super-resolution 3D structured illumination microscopy (3D-SIM ${ }^{24-26}$ ) in mouse embryonic stem cells (ESCs). We used highresolution $\mathrm{Hi}-\mathrm{C}^{27}$ to design pairs of differently labeled Oligopaint probes either within an individual TAD or in two adjacent TADs to compare their intermingling (Fig. 1a, b; Extended Data 1 and 2). The imaging of a probe simultaneously labeled in two colors indicated the precision of our approach (median 3D distance between the centroids of the two segmented colors $=34 \mathrm{~nm}$; Extended Data 3a, b). The quantification of 3D overlap fractions (OFs) revealed a striking higher intermingling of probes located within a TAD (median OF $=0.21 ; 17 \%$ of the cell population displaying an $\mathrm{OF}<0.1$ ) compared to adjacent TADs [median $\mathrm{OF}=0.06 ; 64 \%$ of the cell population displaying an $\mathrm{OF}<0.1$ (Fig. 1c)]. Moreover, for similar genomic distances separating probe centers ( $245 \mathrm{~kb}$ versus $275 \mathrm{~kb}$ ), a much shorter 3D distance distribution was observed within the same TAD compared to between TADs (median distance of $185 \mathrm{~nm}$ versus $349 \mathrm{~nm}$ ), thus reflecting the constrained arrangement of the chromatin fiber within TADs (Fig. 1c). Measurements of the correlation of probe signal intensities, a method free of image-segmentation, confirmed these data (Extended Data 3c). Averaged pictures 
aggregating all individual structures reflect the differential spatial organizations of probes, with those within TAD appearing to belong to the same unit while those between TADs being physically separated (Fig. 1a, averaged panel). We confirmed the higher OFs and shorter 3D distances within TADs than between TADs (Fig. 1e; Extended Data $3 \mathrm{~d}-\mathrm{f}$ ) by extending the analysis to a comprehensive set of 15 probe pairs that were similarly designed to locate either within individual TADs or in two adjacent TADs (Fig. 1d and Extended Data 1). The variability in OFs and distance distributions between the different probe pairs also indicated that TAD structures and insulation do not represent a constant organization but rather vary along the genome, consistent with previous Hi-C measurements ${ }^{28}$. Moreover, the relatively broad distributions observed for both OFs and 3D distances within the same probe pair reflected the cell-to-cell heterogeneity of chromatin folding. Although the intermingling between probes separated by a border often corresponds to limited inter-TAD contacts, a subset of them displayed OF values similar to those measured from probes within TADs (19\% of OFs calculated from probe pairs between TADs surpassed the median OF from probe pairs within TADs; Extended Data 3e), suggesting that merging of adjacent TADs can occur. Yet, in a large majority of single cells, the degree of chromatin intermingling is higher within TADs compared to adjacent TADs. To examine the relationship between Hi-C and FISH, we calculated the fraction of $\mathrm{Hi}-\mathrm{C}$ reads connecting consecutive probes (hereafter defined as crossing contact fraction) for each labeled locus (Extended Data 3g). This score highly correlated with the mean OF $(\rho=0.88 ; p<$ 0.001 ), and inversely correlated with the mean 3D distance between probe centroids $[\rho=-0.85 ; p<$ 0.001 (Fig. 1f)], demonstrating a strong relationship between Hi-C contacts and the physical organization of TADs. This also indicates that super-resolution FISH successfully captures the spectrum of the different interaction strengths measured by cell-population Hi-C. We then investigated the relationship between scHi-C data ${ }^{18}$ and imaging. Confirming previous observations, contacts in scHi-C appeared to vary between cells (Extended Data $3 \mathrm{~h}$ ) ${ }^{17-19}$. Nevertheless, in most cells the crossing contact fraction between labeled regions were significantly higher within TADs than between TADs for which borders were defined by cell-population Hi-C (Extended Data 3i, j). Altogether, these data demonstrate that TADs form heterogeneous chromosomal entities that favor intra-TAD over inter-TAD contacts in individual cells.

ESCs have a plastic chromatin organization that may be reflected in a more cell-to-cell heterogeneous TAD organization compared to differentiated cells ${ }^{27,29}$. To address this, we used an in vitro differentiation system to generate neural progenitor cells (NPCs) from ESCs (Fig. 2a) and utilized Hi$\mathrm{C}$ available from the same cell type ${ }^{27}$. In order to analyze chromatin folding in a pure population of NPCs, we combined FISH with a Pax6 neuronal transcription factor immunostaining (Extended Data 4a). Consistent with the results in ESCs, OFs were higher and physical distances shorter for probes located within individual TADs than between TADs (Fig. 2b; Extended Data 4b, c). Crossing contact 
fractions measured in NPCs were remarkably well correlated with the OFs $(\rho=0.96 ; p<0.001)$, and inversely correlated with the mean physical distances between probe centroids $[\rho=-0.94 ; p<0.001$ (Fig. 2c)]. Moreover, differences in chromatin intermingling within or between TADs were exacerbated in NPCs compared to ESCs, as measured by the increase of the ratio of the mean OFs within TAD over between TADs (Fig. 2d and Extended Data 4d). Consistently, only 9\% of the OFs between TADs surpassed the median OF measured from probes within TADs in NPCs. The spatial segregation of chromatin into TADs is therefore enhanced in NPCs, in accordance with increased insulation observed in Hi-C ${ }^{27,29}$. Furthermore, probes in NPCs were globally more condensed than in ESCs (Extended Data 4e). Nevertheless, dispersion measurements for both OFs and 3D distances indicated similar relative cell-to-cell variability in TAD intermingling between the two cell types, indicating that cell heterogeneity persists during differentiation (Extended Data 4f). We then studied regions that are structurally rewired during differentiation, such as distinct TADs in ESCs merging into one in NPCs [pairs of probes 21-22, 32-33, 101-103 (Extended Data 1)]. At the zfp42 locus, the border between two TADs in ESCs, associated with high expression of the $z f p 42$ gene, becomes abolished in NPCs upon repression of the gene (Fig. 2e) ${ }^{27}$. The disappearance of the border corresponds to a fusion of the two genomic regions into a larger domain with higher intermingling (ESCs versus NPCs, median OFs $=0.04$ and 0.19, median 3D distances between centroids $=490$ and $295 \mathrm{~nm}$, respectively; Fig. 2f, g), confirming the physical nature of the insulation detected by Hi-C (Extended Data 4g). A similar structural organization of this locus was observed in vivo in mouse E14.5 neocortex NPCs (ncxNPCs) (Fig. 2e-g; Extended Data Fig. 4g). We also investigated a situation where a weak internal border is formed in the absence of CTCF at the transcription start site of the activated zfp608 gene in NPCs (Extended Data $4 \mathrm{~h}$ ) ${ }^{27}$. In this case and in agreement with the crossing contact fraction measured in Hi-C, no significant changes in OFs and 3D distances were observed between cell types (Extended Data 4i-k), consistent with an internal transcription-associated rewiring of contacts inside the original TAD instead of a split into two distinct physically insulated domains.

CTCF and cohesin were previously shown to be essential for TAD separation in Hi-C 11,13,15,16. However, Hi-C does not provide information on the physical nature of the loss of insulation between TADs in individual CTCF- and cohesin-depleted cells. We thus performed FISH and super-resolution imaging in a transgenic mouse embryonic stem cell line (referred to as CTCF-AID) in which endogenous CTCF can be degraded upon auxin treatment (Extended Data 5a) ${ }^{11}$. Upon CTCF depletion, TAD-dependent differences in chromatin intermingling were totally abolished, such that OFs and 3D distances between intra- or inter-TAD probes were very similar (Fig. 3a-d and Extended Data $5 \mathrm{~b}, \mathrm{c} ; 52 \%$ of the OFs between TADs surpassed the median OF measured from probes within TADs). Loss of TAD borders induced ectopic intermingling between previously insulated regions 
(mean inter-TAD OF increase of $38 \%$ compared to untreated cells), showing that CTCF prevents contacts between adjacent TADs (Fig. 3c, e and Extended Data 5b). We also observed a decrease in intra-TAD interactions (mean intra-TAD OF decrease of 14\%; Fig. 3e and Extended Data 5b), indicating a partial redistribution of chromatin contacts across TAD borders. The spatial segregation of TADs in CTCF-AID untreated cells was already weaker than in wild-type ESCs (Fig 2d and Extended Data 5c), in agreement with the previously reported 2- to 3-fold decrease of CTCF level in untreated CTCF-AID compared to wild-type cells ${ }^{11}$. To compare the roles of CTCF and cohesin in TAD formation, we next generated a mouse embryonic stem cell line in which an AID tag was introduced into the RAD21 cohesin subunit (RAD21-AID) to induce its depletion upon auxin treatment (Extended Data 5d). In RAD21-depleted cells, differences in intra- versus inter-TAD chromatin intermingling were also strongly reduced (Fig. 3a-c, f and Extended Data 5e, f; 43\% of the OFs between TADs surpassed the median OF measured from probes within TADs). However, in contrast to CTCF depletion, the loss of preferential contacts within TADs was due to a decrease of intra-TAD interactions (mean intra-TAD OF decrease of 41\%), while ectopic intermingling between adjacent TADs was generally not observed (mean inter-TAD OF increase of $6 \%$ ), strongly suggesting that the primary function of cohesin is to generate chromatin contacts (Fig. 3c, g and Extended Data 5e). Consistent with the unfolding of TADs upon RAD21 depletion, 3D distances between probe centroids located in adjacent TADs were larger than in untreated cells (Fig. 3c and Extended Data 5e). Therefore, the enrichment of chromosomal contacts within TADs is regulated by the combined action of CTCF and cohesin through distinct mechanisms: cohesin is responsible for chromatin contacts that generate intermingling of distant chromatin regions, while CTCF mainly prevents inter-TAD contacts.

We further utilized super-resolution FISH to characterize inner TAD structural features that cannot be assessed with diffraction-limited microscopy (Extended Data 6a-c). TADs range from condensed spherical structures to stretched and decondensed conformations (Extended Data 6d). Furthermore, they are organized into smaller discrete chromatin nanodomains (CNDs) that vary in size and number within each TAD (Fig. 4a, b; Extended Data 6e, f). DAPI-stained nuclei that were not submitted to FISH confirmed the folding of the chromatin into CNDs with sizes in good agreement with those measured within TADs (median CND size of $235 \mathrm{~nm}$ and $247 \mathrm{~nm}$ for DAPI and for TADs labeled by FISH, respectively; Fig. 4c, d and Extended Data 6g). Of note, CNDs were readily detected even in probes homogeneously covering regions with uniform epigenetic features that do not display internal borders in Hi-C despite very high sequencing depth (Fig. 4a and Extended Data 1 and 2). This indicates that cell-to-cell variable boundaries prevent their identification in ensemble Hi-C experiments. Therefore, CNDs represent a general organization of chromatin rather than the previously described "sub-TADs", which are detected by Hi-C or micro-C 12,30,31 within TADs characterized by specific epigenomic composition or by CTCF sites. Unlike TAD insulation, TAD 
structures and CNDs were largely unaffected upon CTCF degradation (Extended Data 7a, b). In RAD21-depleted cells, we observed an increase in TAD volume and in the number of CNDs per TAD (Extended Data 7c). However, a previous study using similar cohesin depletion experiments in ESCs showed that it induces defects in cell cycle progression with a shift from G0/G1 to G2/M phases ${ }^{32}$. Moreover, our imaging data revealed that individual alleles in interphase nuclei often appeared as two distinct segmented objects, indicating that replicated sister chromatids were improperly paired upon RAD21 removal (Extended Data 7d). Individual unpaired sister chromatids in RAD21-depleted cells and individual chromosomes in G1-staged ESCs showed a similar folding into TADs and CNDs (Extended Data 7e, f). The observed changes in TAD volumes and shapes and in CND numbers under RAD21 depletion are thus mainly due to replicated sister chromatids adopting a loose pairing configuration. Furthermore, the size of CNDs remained mostly unaffected (Extended Data 7c). Therefore, the organization of chromosomes into CNDs persists in cells depleted for CTCF or cohesin, consistent with CNDs and TADs representing two distinct layers of chromosome folding. On the other hand, we observed that inactive chromatin regions were more condensed and folded into fewer CNDs than active ones (Extended Data 8a-f). We therefore hypothesized that the formation of CNDs depend on nucleosome-nucleosome interactions. Since histone acetylation is known to prevent these interactions ${ }^{6,33}$, we treated ESCs with trichostatin-A (TSA), an inhibitor of histone deacetylases which induces histone hyper-acetylation (Extended Data 8g). In TSA-treated ESCs, TADs remained spatially segregated (Extended Data 8h, i). However, histone hyper-acetylation disrupted CND organization, leading to a decrease in their volume associated with an increase in their number (Fig. 4e-g; Extended Data 8j). Hence, the formation of CNDs relates to the chromatin state. Finally, we previously reported the organization of Drosophila chromosomes into discrete chromatin domains ${ }^{20}$. In this species, TADs do not appear to rely on CTCF and cohesin ${ }^{34}$, and have a smaller genomic size compared to their mammalian counterpart (mean size $\sim 100 \mathrm{~kb}$ versus $\sim 900 \mathrm{~kb}$ ) ${ }^{3,4,35}$. Interestingly, the size and structure of a $110 \mathrm{~kb}$ Drosophila TAD closely resembles CNDs (Extended Data 6h, i), suggesting that, in this species, both correspond to the same structural layer of chromatin folding, as opposed to mouse in which TADs generated by CTCF and cohesin encompass several CNDs (Fig. 4b, d).

\section{Discussion}

By applying super-resolution microscopy to image many loci in different cell types, we were able to characterize the fine structural organization of TADs as well as their relative spatial organization in individual cells (Fig. 4h). Our data indicate that heterogeneous domains with various shapes and resilient borders can coexist with enriched intra-TAD interactions, reconciling the variable nature of TADs detected by scHi-C ${ }^{17-19}$ and microscopy ${ }^{21,36}$ and their potential to form regulatory landscapes ${ }^{8}$. Moreover, the characterization of the structural organization of chromosomes both in CTCF- and 
RAD21- depleted cells allowed us to discriminate their role in shaping TADs. Cohesin generates chromatin intermingling, while CTCF, by defining TAD borders, delimitates cohesin-induced chromatin interactions within TADs, in line with the loop-extrusion mechanism ${ }^{9,14,37,38}$. These results are also consistent with a recent study showing that cohesin promotes domain intermingling ${ }^{39}$. Finally, the identification of an intermediate level of physical folding between nucleosomes and TADs represented by discrete and heterogeneous CNDs is consistent with super-resolution live-imaging analyses ${ }^{40}$. Here, we show that this structural organization is distinct from TADs in mammals (Fig. 4h), clarifying the observation of globular domains in single cells with physical insulations located outside CTCF borders that persist after removal of cohesin ${ }^{21}$. Previous studies showed that nucleosomes can form clutches that depend on their acetylation states $5,6,33$, suggesting that CNDs contain ensembles of smaller clutches that aggregate by a self-assembly mechanism driven by nucleosome interactions ${ }^{41}$. It will be important to elucidate the functional role of CNDs, whether CNDs might constrain enhancer-promoter interactions (which are in the same $\sim 100 \mathrm{~kb}$ range as the size of CNDs), and to investigate mechanisms that regulate the formation, size and topological consequences of CNDs and TADs on vertebrate genomes.

\section{Acknowledgements}

We thank R. Saldana-Meyer for sharing the CTCF-AID cell line. We thank the Montpellier Resources Imagerie facility (BioCampus Montpellier, CNRS, INSERM, University of Montpellier) and J. Mateos-Langerak for microscopy support. We thank L. Fritsch for help in Western Blot. Funding: Q.S. was supported by the French Ministry of Higher Education and Research and la Ligue Nationale Contre le Cancer. A.D. was supported by the European Research Council (Advanced Grant 3DEpi). I.J. was supported by EMBO Long-Term Fellowship (ALTF 559-2018) and the Laboratory of Excellence EpiGenMed. G.L.P. was supported by a grant from the Agence Nationale de la Recherche (ANR-18-CE15-010). T.C. was supported by CNRS. B.B. was supported by a Sir Henry Wellcome Postdoctoral Fellowship 100136MA/Z/12/Z. B.G.B. was supported by grants from the NIH/NHLBI (P01HL089707, Bench to Bassinet Program UM1HL098179). F.B. and G.C. were supported by CNRS. Research in the G.C. laboratory was supported by grants from the European Research Council (Advanced Grant 3DEpi, under grant agreement No 788972), the European Union's Horizon 2020 research and innovation programme (MuG, under grant agreement No 676556), the Agence Nationale de la Recherche (ANR-15-CE12-0006 EpiDevoMath), the Fondation pour la Recherche Médicale (DEI20151234396), the MSDAVENIR foundation (Project GENE-IGH), the INSERM and the French National Cancer Institute (INCa) . 
240 Q.S., F.B., and G.C. initiated and led the project. Q.S. and F.B. designed Oligopaint probes. Q.S. and A.D. produced Oligopaint probes. Q.S. performed FISH experiments, 3D-SIM image acquisition and image analysis. Q.S., A.D. and I.J. handled ESC culture. A.D. and I.J. handled CTCF-AID and RAD21-AID culture and performed NPC differentiation. A.D. performed western blots. G.L.P analyzed sequencing data. T.C. performed STED imaging. B.B. dissected mouse neocortices. E.N. and B.G.B. generated the CTCF-AID and RAD21-AID cell lines. F.B. handled flies. Q.S., F.B., and G.C. wrote the manuscript. All the authors discussed the data and reviewed the manuscript.

\section{Competing interest statement}

The authors declare no competing interests.

\section{Main references}

Dixon, J. R. et al. Topological domains in mammalian genomes identified by analysis of chromatin interactions. Nature 485, 376-380, doi:10.1038/nature11082 (2012).

2 Hou, C., Li, L., Qin, Z. S. \& Corces, V. G. Gene density, transcription, and insulators contribute to the partition of the Drosophila genome into physical domains. Mol Cell 48, 471-484, doi:10.1016/j.molcel.2012.08.031 (2012).

3 Nora, E. P. et al. Spatial partitioning of the regulatory landscape of the X-inactivation centre. Nature 485, 381-385, doi:10.1038/nature11049 (2012).

4 Sexton, T. et al. Three-Dimensional Folding and Functional Organization Principles of the Drosophila Genome. Cell 148, 458-472, doi:10.1016/j.cell.2012.01.010 (2012).

Ou, H. D. et al. ChromEMT: Visualizing 3D chromatin structure and compaction in interphase and mitotic cells. Science 357, doi:10.1126/science.aag0025 (2017).

6 Ricci, M. A., Manzo, C., Garcia-Parajo, M. F., Lakadamyali, M. \& Cosma, M. P. Chromatin fibers are formed by heterogeneous groups of nucleosomes in vivo. Cell 160, 1145-1158, doi:10.1016/j.cell.2015.01.054 (2015).

7 Cremer, T. \& Cremer, M. Chromosome territories. Cold Spring Harbor perspectives in biology 2, a003889, doi:10.1101/cshperspect.a003889 (2010).

8 Robson, M. I., Ringel, A. R. \& Mundlos, S. Regulatory Landscaping: How Enhancer-Promoter Communication Is Sculpted in 3D. Mol Cell 74, 1110-1122, doi:10.1016/j.molcel.2019.05.032 (2019).

9 Fudenberg, G. et al. Formation of Chromosomal Domains by Loop Extrusion. Cell reports, doi:10.1016/j.celrep.2016.04.085 (2016).

10 Haarhuis, J. H. I. et al. The Cohesin Release Factor WAPL Restricts Chromatin Loop Extension. Cell 169, 693-707 e614, doi:10.1016/j.cell.2017.04.013 (2017).

11 Nora, E. P. et al. Targeted Degradation of CTCF Decouples Local Insulation of Chromosome Domains from Genomic Compartmentalization. Cell 169, 930-944 e922, doi:10.1016/j.cell.2017.05.004 (2017).

12 Rao, S. S. et al. A 3D map of the human genome at kilobase resolution reveals principles of chromatin looping. Cell 159, 1665-1680, doi:10.1016/j.cell.2014.11.021 (2014). 
Rao, S. S. P. et al. Cohesin Loss Eliminates All Loop Domains. Cell 171, 305-320.e324, doi:10.1016/j.cell.2017.09.026 (2017).

14 Sanborn, A. L. et al. Chromatin extrusion explains key features of loop and domain formation in wildtype and engineered genomes. Proc Natl Acad Sci U S A, doi:10.1073/pnas.1518552112 (2015).

15 Schwarzer, W. et al. Two independent modes of chromatin organization revealed by cohesin removal. Nature 551, 51-56, doi:10.1038/nature24281 (2017).

16 Wutz, G. et al. Topologically associating domains and chromatin loops depend on cohesin and are regulated by CTCF, WAPL, and PDS5 proteins. EMBO J 36, 3573-3599, doi:10.15252/embj.201798004 (2017).

17 Flyamer, I. M. et al. Single-nucleus Hi-C reveals unique chromatin reorganization at oocyte-to-zygote transition. Nature, doi:10.1038/nature21711 (2017).

18 Nagano, T. et al. Cell-cycle dynamics of chromosomal organization at single-cell resolution. Nature 547, 61-67, doi:10.1038/nature23001 (2017).

19 Stevens, T. J. et al. 3D structures of individual mammalian genomes studied by single-cell Hi-C. Nature, doi:10.1038/nature21429 (2017).

20 Szabo, Q. et al. TADs are 3D structural units of higher-order chromosome organization in Drosophila. Science advances 4, eaar8082, doi:10.1126/sciadv.aar8082 (2018).

21 Bintu, B. et al. Super-resolution chromatin tracing reveals domains and cooperative interactions in single cells. Science 362, doi:10.1126/science.aau1783 (2018).

22 Beliveau, B. J. et al. Single-molecule super-resolution imaging of chromosomes and in situ haplotype visualization using Oligopaint FISH probes. Nature communications 6, 7147, doi:10.1038/ncomms8147 (2015).

23 Beliveau, B. J. et al. OligoMiner provides a rapid, flexible environment for the design of genome-scale oligonucleotide in situ hybridization probes. Proc Natl Acad Sci U S A 115, E2183-E2192, doi:10.1073/pnas.1714530115 (2018).

24 Demmerle, J. et al. Strategic and practical guidelines for successful structured illumination microscopy. Nature protocols 12, 988-1010, doi:10.1038/nprot.2017.019 (2017).

25 Gustafsson, M. G. et al. Three-dimensional resolution doubling in wide-field fluorescence microscopy by structured illumination. Biophys J 94, 4957-4970, doi:10.1529/biophysj.107.120345 (2008).

26 Markaki, Y. et al. The potential of 3D-FISH and super-resolution structured illumination microscopy for studies of 3D nuclear architecture: 3D structured illumination microscopy of defined chromosomal structures visualized by 3D (immuno)-FISH opens new perspectives for studies of nuclear architecture. Bioessays 34, 412-426, doi:10.1002/bies.201100176 (2012).

27 Bonev, B. et al. Multiscale 3D Genome Rewiring during Mouse Neural Development. Cell 171, 557572.e524, doi:10.1016/j.cell.2017.09.043 (2017).

28 Zhan, Y. et al. Reciprocal insulation analysis of $\mathrm{Hi}-\mathrm{C}$ data shows that TADs represent a functionally but not structurally privileged scale in the hierarchical folding of chromosomes. Genome Res 27, 479-490, doi:10.1101/gr.212803.116 (2017).

29 Barrington, C. et al. Enhancer accessibility and CTCF occupancy underlie asymmetric TAD architecture and cell type specific genome topology. Nature communications 10, 2908, doi:10.1038/s41467-01910725-9 (2019).

30 Hsieh, T. S. et al. Resolving the 3D Landscape of Transcription-Linked Mammalian Chromatin Folding. Mol Cell, doi:10.1016/j.molcel.2020.03.002 (2020).

31 Krietenstein, N. et al. Ultrastructural Details of Mammalian Chromosome Architecture. Mol Cell, doi:10.1016/j.molcel.2020.03.003 (2020).

32 Rhodes, J. D. P. et al. Cohesin Disrupts Polycomb-Dependent Chromosome Interactions in Embryonic Stem Cells. Cell reports 30, 820-835.e810, doi:10.1016/j.celrep.2019.12.057 (2020).

33 Otterstrom, J. et al. Super-resolution microscopy reveals how histone tail acetylation affects DNA compaction within nucleosomes in vivo. Nucleic Acids Res 47, 8470-8484, doi:10.1093/nar/gkz593 (2019).

34 Matthews, N. E. \& White, R. Chromatin Architecture in the Fly: Living without CTCF/Cohesin Loop Extrusion?: Alternating Chromatin States Provide a Basis for Domain Architecture in Drosophila. Bioessays 41, e1900048, doi:10.1002/bies.201900048 (2019).

35 Szabo, Q., Bantignies, F. \& Cavalli, G. Principles of genome folding into topologically associating domains. Science advances 5, eaaw1668, doi:10.1126/sciadv.aaw1668 (2019). 
Finn, E. H. et al. Extensive Heterogeneity and Intrinsic Variation in Spatial Genome Organization. Cell 176, 1502-1515 e1510, doi:10.1016/j.cell.2019.01.020 (2019).

37 Davidson, I. F. et al. DNA loop extrusion by human cohesin. Science 366, 1338-1345, doi:10.1126/science.aaz3418 (2019).

38 Kim, Y., Shi, Z., Zhang, H., Finkelstein, I. J. \& Yu, H. Human cohesin compacts DNA by loop extrusion. Science 366, 1345-1349, doi:10.1126/science.aaz4475 (2019).

39 Luppino, J. M. et al. Cohesin promotes stochastic domain intermingling to ensure proper regulation of boundary-proximal genes. Nat Genet, doi:10.1038/s41588-020-0647-9 (2020).

40 Nozaki, T. et al. Dynamic Organization of Chromatin Domains Revealed by Super-Resolution Live-Cell Imaging. Mol Cell 67, 282-293 e287, doi:10.1016/j.molcel.2017.06.018 (2017).

41 Kantidze, O. L. \& Razin, S. V. Weak interactions in higher-order chromatin organization. Nucleic Acids Res 48, 4614-4626, doi:10.1093/nar/gkaa261 (2020).

\section{Figures legends}

Fig. 1 | TADs form heterogeneous structures in single cells, favoring intra-TAD over inter-TAD chromatin intermingling. a, Left: Hi-C maps from ESCs along with probe locations, either within a TAD (top) or between two adjacent TADs (bottom). Right: representative 3D-SIM images of the probes shown in a and averaged images generated from all segmented probe pairs (48 and 50 alleles were analyzed for the pair 51a-51b and 51b-52, respectively). Maximum projections, scale bars: $500 \mathrm{~nm}$. b, 3D-views from different individual segmented probe pairs shown in a. c, Overlap fractions (OFs) and 3D distances between centroids of the probes shown in a. Boxplots represent median, interquartile ranges and Tukey-style whiskers. $\mathrm{n}=48$ and 50 for the pair 51a-51b and 51b-52, respectively; ***, $p<0.001$; two-sided Wilcoxon rank sum tests. d, Experimental design to label individual or adjacent TADs. e, OFs and 3D distances between centroids measured for each probe pair as a function of the genomic distance separating their centers. Graph represents medians and interquartile ranges. A mean of 65 alleles was analyzed per probe pair. f, OFs and 3D distances between centroids as a function of crossing contact fraction measured in $\mathrm{Hi}-\mathrm{C}$ along with the goodness of fit $\left(\mathrm{R}^{2}\right)$ of a linear regression and the Pearson's correlation coefficient $\rho$.

Fig. 2 | The spatial segregation of TADs increases during cell differentiation. a, NPCs were generated from in vitro differentiation of ESCs. b, OFs and 3D distances between centroids measured for each probe pair in NPCs as a function of the genomic distance separating their centers. Graph represents medians and interquartile ranges. A mean of 36 alleles was analyzed per probe pair. c, OFs and 3D distances between centroids as a function of crossing contact fraction measured in Hi-C along with the goodness of fit $\left(\mathrm{R}^{2}\right)$ of a linear regression and the Pearson's correlation coefficient $\rho$. d, Mean OF measured from all probe pairs within TADs divided by 
the mean OF from all probe pairs between TADs (using probe pairs that do not change their TAD borders between ESCs and NPCs). e, Hi-C maps from ESCs, NPCs, and ncxNPCs along with probe pair location. Arrowhead indicates the location of the expressed $z f p 42$ gene. f, Representative 3D-SIM images of the probes shown in e (50, 26 and 18 alleles were analyzed in ESCs, NPCs, ncxNPCs, respectively). Maximum projections, scale bar: $500 \mathrm{~nm}$. g, OFs and 3D distances between the centroids of the probes shown in e. Boxplots represent median, interquartile ranges and Tukey-style whiskers. $\mathrm{n}=50,26$ and 18 for ESCs, NPCs, and ncxNPCs, respectively; ***, $p<0.001 ; * *, p<0.01$ * $^{*}, p<0.05$; two-sided Wilcoxon rank sum tests.

Fig. 3 | Cohesin generates chromatin intermingling while CTCF prevents inter-TAD contacts. a, Hi-C maps from ESCs along with probe locations, either between two adjacent TADs (probe pair 101-102a) or within a TADs (probe pair 102a-102b). b, Representative 3D-SIM images of the probes shown in a in the indicated cell types (probe pairs 101-102a/102a-102b; 52/58, 47/57, 163/130 and 54/61 alleles were analyzed in CTCF-AID, CTCF-AID + auxin, RAD21-AID and RAD21-AID + auxin, respectively). Maximum projections, scale bar: $500 \mathrm{~nm}$. c, OFs and 3D distances between the centroids of the probes shown in a. Boxplots represent median, interquartile ranges and Tukey-style whiskers. $\mathrm{n}$ (probe pairs 101-102a/102a-102b) = 52/58, 47/57, 163/130 and 54/61 for CTCF-AID, CTCF-AID + auxin, RAD21-AID and RAD21-AID + auxin, respectively; ***, $p<0.001 ; * *, p<0.01 ; *, p<0.05$; two-sided Wilcoxon rank sum tests. d, OFs and 3D distances between centroids measured for each probe pair in CTCF-AID + auxin cells as a function of the genomic distance separating their centers. Graph represents medians and interquartile ranges. A mean of 51 alleles was analyzed per probe pair. e, Mean ( \pm standard deviation "SD") OF fold change (CTCF-AID + auxin / CTCF-AID) for probe pairs within TADs $(\mathrm{n}=7)$ or between TADs $(\mathrm{n}=8) ; * *, p=0.0019 ;^{*} p=0.016$; two-sided t-test. f, OFs and 3D distances between centroids measured for each probe pair in RAD21-AID + auxin cells as a function of the genomic distance separating their centers. Graph represents medians and interquartile ranges. A mean of 47 alleles was analyzed per probe pair. g, Mean $( \pm \mathrm{SD})$ OF fold change (RAD21-AID + auxin / RAD21-AID) for probe pairs within TADs $(\mathrm{n}=7)$ or between TADs $(\mathrm{n}=8) ; * *, p=0.0012$; two-sided t-test.

Fig. 4 | TADs are subdivided into discrete chromatin nanodomains (CNDs) that depend on histone acetylation. a, Hi-C map from ESCs along with probe location (TAD \#22) and ChIP-seq tracks. b, Top: representative 3D-SIM images of the TAD shown in a (51 alleles were analyzed). White lines represent the boundaries probe segmentations ( $2 \mathrm{D}$ projections). Maximum projections, scale bar $=500 \mathrm{~nm}$. Bottom: $3 \mathrm{D}$ views of the segmented TADs shown above (gray mesh) and watershed segmented CNDs (colored objects). c, DAPI staining in ESC ( 3 nuclei were analyzed). Single $z$-slice, scale bars $=5 \mu \mathrm{m}$ and $500 \mathrm{~nm}$ in the magnification. d, Extrapolated diameters (diameter of a circle with the same area than the segmented object) of TADs (ranging from 215 to $990 \mathrm{~kb}$ ), of CNDs within them, and of CNDs measured with DAPI staining. Bins represent $50 \mathrm{~nm}, \mathrm{n}=804,1413$ and 3068, respectively. e, Representative 3D-SIM images of TAD \#62 in ESC and ESC + TSA (94 and 74 alleles were analyzed in ESCs and ESCs + TSA, respectively). White lines represent the boundaries of probe segmentations (2D projections). Maximum projections, scale bar $=500 \mathrm{~nm}$. $\mathbf{f}$, 
CND volumes. Boxplots represent median, interquartile ranges and Tukey-style whiskers. A mean of 296 and 417 CNDs was analyzed per probe in ESCs and ESCs + TSA, respectively; ***, $p<0.001$; **, $p<0.01 ; *, p<$ 0.05 ; two-sided Wilcoxon rank sum tests. g, Mean ( \pm SD) number of CNDs per TAD. A mean of 93 and 95 alleles was analyzed per probe in ESCs and ESCs + TSA, respectively; ***, $p<0.001 ; * *, p<0.01$; two-sided Wilcoxon rank sum tests. h, Model of 3D TAD folding. TADs, which form variable structures and are subdivided into smaller CNDs, favor chromatin intermingling in most cells. Their spatial segregation is further exacerbated in differentiated NPCs. Upon CTCF depletion, the cohesin complex can extrude chromatin ${ }^{37,38}$ through TAD borders, inducing ectopic contacts between adjacent TADs and abolishing preferential intra-TAD interactions. Upon RAD21 depletion, preferential intra-TAD interactions are lost due to the absence of intermingling generated by the cohesin complex. Upon TSA-mediated histone hyper-acetylation, TADs remain spatially segregated while the structural organization of CNDs is disrupted.

\section{Tables}

There are no tables in the present study.

\section{Methods}

\section{Cell culture and tissue preparation}

To generate the RAD21-AID cell line, E14Tg2a cells were transfected using a Neon transfection system, using one million cells and $15 \mu \mathrm{g}$ of a Rad21-AID-eGFP targeting vector (pEN527, Addgene \# 156452) together with $5 \mu \mathrm{g}$ of a pX330-derived vector encoding Cas9 and a sgRNA against CCACGGTTCCATATTATCTG (pX330-EN1082, Addgene \#156450). Cells were selected with blasticidin as a pool for 10 days and transfected with pCAGGS-Flpo-IRES-puro and subcloned without antibiotics. A homozygous blasticidin-sensitive clone was identified by PCR and used to knock in a Tir1-2A-puro cassette at the Tigre locus using Addgene 92142 with 92144 and subcloned with puromycin, yielding cell line EN272.2.

ESCs (E14Tg2a cell line) were cultured on 0.1\% gelatin (G1890-100G, Sigma-Aldrich) coated dishes in GMEM (21710025, Gibco), supplemented with 15\% FBS qualified serum (6140079, Thermofisher Scientific, USA origin), $1 \times$ Glutamax (35050038, Thermofisher Scientific), $1 \times$ MEM Non-Essential Amino Acids (11140035, Thermofisher Scientific), 50 U Penicillin-Streptomycin (15140122, Gibco), $0.1 \mathrm{mM}$ Sodium Pyruvate (11360070, Gibco), $0.1 \mathrm{mM}$ 2-Mercaptoethanol (31350010, Gibco), and 1000 U/mL LIF (ESG1107, Sigma-Aldrich). Media was changed every day and cells were passaged every two days using TrypLE Express Enzyme (12604013, Gibco). Histone hyper-acetylation was 
performed by incubating ESCs with TSA (647925, Sigma-Aldrich, $100 \mathrm{ng} / \mathrm{mL}$ final concentration) for 6 hours before harvesting. CTCF-AID cells (ID\#EN204.3) ${ }^{42}$ were cultured and induced as previously described in ${ }^{11}$. RAD21-AID cells were cultured without Penicillin-Streptomycin using the media previously described for ESCs. Briefly, Indole-3-Acetic Acid sodium salt (16954, Auxin analog, Cayman) was added in the media (final concentration at $500 \mu \mathrm{M}$ ) for 2 days or 6 hours before harvesting CTCF-AID or RAD21-AID cells, respectively.

ESCs were differentiated into NPCs as described in ${ }^{43}$ with the following changes. Cells were plated $\left(2.5 \times 10^{5}\right.$ cells per $10 \mathrm{~cm}$ dish) on $0.1 \%$ gelatin-coated dishes in the ESC media previously described. After 1 day, cells were cultured in DDM media (DMEM/F-12, GlutaMAX Supplement; 31331-028, Gibco), supplemented with 0.05\% BSA (15260-037, Gibco), 1× MEM Non-Essential Amino Acids (11140035, Thermofisher Scientific), 50 U Penicillin-Streptomycin (15140122, Gibco), 1× N-2 Supplement (17502-048, Gibco), 1 mM Sodium Pyruvate (11360070, Gibco) and $0.1 \mathrm{mM} 2-$ Mercaptoethanol (31350010, Gibco). From day 2 to day 10, media was changed every 2 days and cyclopamine (239803-1MG, Millipore) was added in the media (final concentration at $0.4 \mu \mathrm{g} / \mathrm{mL}$ ). After 12 days of differentiation, cells were dissociated with StemPro Accutase (A11105-01, Gibco), plated on 6-well plates coated with Poly-L-lysine (P2636, Sigma)/Laminin (11243217001, Sigma), and cultured in a 1:1 mixture of DDM and Neurobasal/N27 media [(21103049, Gibco) supplemented with 1× B27 without vitamin A (12587-010, Gibco) and 1× GlutaMAX Supplement (31331-028, Gibco)] and 50 U Penicillin-Streptomycin (15140122, Gibco) for additional 2 days (D12+2) to obtain NPCs.

Brains from E14.5 C57BL/6J embryos were dissected and fixed in 4\% PFA overnight at $4^{\circ} \mathrm{C}$. They were then cryopreserved for $24 \mathrm{~h}$ at $4{ }^{\circ} \mathrm{C}$ in a PBS-20\% sucrose solution. After embedding in OCT and fast freezing in isopentane, brains were sectioned at $10 \mu \mathrm{m}$ width on slides with a cryostat (Leica). All mice were housed and maintained following the guidelines of the Animal Care and Use Committee of the Institut National de la Santé et de la Recherche Médicale (INSERM) in accordance with the European Council directive (2010/63/UE) for the protection and use of vertebrate animals.

Flies were raised in standard cornmeal yeast extract media at $21^{\circ} \mathrm{C}$. Embryos were harvested on agar/vinegar plate at stages 15 to 16 after egg laying, equivalent to a development of 12 to 16 hours at $25^{\circ} \mathrm{C}$. Embryos were manually dechorionated on a double-face adhesive tape and displayed on an agar/vinegar plate to avoid drying during manual selection under a GFP binocular. To analyze the 110 kb Drosophila TAD in an haploid context, i.e. on chromosome $\mathrm{X}$ in male embryos, a Y-GFP reporter line (y[1], w[67c23];Dp(1;Y), y[+] P $\{\mathrm{ry}+11\} \mathrm{P}\{\mathrm{w}[+\mathrm{mC}]=$ ActGFP $\}$ JMR1) was used for the selection of WT GFP+ male embryos (Szabo et al, 2018). 


\section{Western blot}

481

482

483

484

485

486

487

488

489

490

491

492

493

494

495

496

497

498

499

500

501

502

503

504

505

506

507

508

509

510

511

512

For CTCF Western-Blot, ESCs (approximately $10 \times 10^{6}$ cells) were dissociated, resuspended in cold PBS, pelleted and resuspended in $10 \mathrm{mM}$ HEPES-KOH pH 7.9, $1.5 \mathrm{mM} \mathrm{MgCl} 2,10 \mathrm{mM} \mathrm{KCl}, 0.5 \mathrm{mM}$ DTT, 0.2 mM PMSF, Protease Inhibitor Cocktail (PIC, 04693132001, Roche; $10 \mu \mathrm{L}$ per 1mL) and kept on ice for 10 minutes. Cells were busted by 10 seconds vortex, centrifuged, resuspended in 20 mM HEPES-KOH pH 7.9, 25\% Glycerin, $420 \mathrm{mM} \mathrm{NaCl}, 1.5 \mathrm{mM} \mathrm{MgCl} 2,0.5 \mathrm{mM}$ DTT, $0.2 \mathrm{mM}$ PMSF, PIC and incubated for 20 minutes on ice. After centrifugation $2 \mathrm{~min}$ at $13,000 \mathrm{rpm}$ at $4^{\circ} \mathrm{C}$, the supernatant was stored at $-80^{\circ} \mathrm{C}$. Protein concentration was measured using Pierce BCA Protein Assay Kit (23227, Thermo Scientific) and 10 or $20 \mu \mathrm{g}$ of nuclear extracts were used per lane and mixed with 4X Bolt LDS Sample Buffer (B0007, Invitrogen) and 10X Bolt ${ }^{\mathrm{TM}}$ Sample Reducing Agent (B0009, Invitrogen) before loading. Acid extraction were performed for histone Western-Blot. Briefly, ESCs (approximately $10 \times 10^{6}$ cells) were dissociated, washed twice in PBS, $5 \mathrm{mM}$ Na Butyrate, pelleted and resuspended in $10 \mathrm{mM}$ HEPES-KOH pH 7.9, $1.5 \mathrm{mM} \mathrm{MgCl2,} 10 \mathrm{mM} \mathrm{KCl,} 0.5 \mathrm{mM}$ DTT, 0.5\% NP40, $5 \mathrm{mM}$ Na Butyrate, PIC (5 volumes of pellet). Cells were lysed by vortex and pipetting. After addition of $\mathrm{H} 2 \mathrm{SO} 4$ at $0.4 \mathrm{~N}$ final, incubation was performed 30 minutes on ice. After centrifugation 15 min at $15,000 \mathrm{rpm}$ at $4^{\circ} \mathrm{C}$, supernatant was collected and 4 volumes of cold acetone was added, followed by an overnight precipitation at $-20^{\circ} \mathrm{C}$. After centrifugation $15 \mathrm{~min}$ at $15,000 \mathrm{rpm}$ at $4^{\circ} \mathrm{C}$, pellets were washed twice in $1 \mathrm{~mL}$ cold acetone and dried $1 \mathrm{~min}$ at $96^{\circ} \mathrm{C}$. The pellet was resuspended in $200 \mu \mathrm{L}$ Sample Buffer and 7.5 or $15 \mu \mathrm{l}$ were loaded per lane. Samples were run on Bolt ${ }^{\mathrm{TM}} 4-12 \%$ Bis-Tris Plus Gels (NW04122BOX, Invitrogen). The transfer was performed for $2 \mathrm{~h}$ at $1 \mathrm{~A}$ with Nitrocellulose/Filter Paper Sandwiches (1620215, Biorad). Membranes were incubated in 10\% milk powder/TBST (TBS/0.2\% Tween 20) for 1 hour at room temperature under gentle agitation. AntiCTCF (61311, Active motif; 1/1000 dilution), anti-RAD21 (ab154769, Abcam; 1/1000 dilution), antiVinculin (sc-73614, SantaCruz Biotechnology; 1/1000 dilution), anti-Histone H4 (Sigma-Aldrich 07108; 1/2000 dilution) or anti-acetyl-Histone H4 (06-598, Sigma-Aldrich; 1/1000 dilution) primary antibodies were incubated overnight in $1 \%$ milk powder/TBST at $4{ }^{\circ} \mathrm{C}$ or 1 hour at room temperature under gentle agitation. After 4 washes in TBST, secondary antibody [Anti-Rabbit IgG-Peroxidase antibody (A0545-1ML, Sigma-Aldrich; 1/15,000 dilution), or Anti-Mouse IgG-Peroxidase antibody (A9044-2ML; 1/15,000 dilution) was incubated for 1 hour at room temperature under gentle agitation. Before Vinculin staining, the membrane was stripped with Restore ${ }^{\mathrm{TM}}$ Western Blot Stripping Buffer (21059, Thermo Scientific). Revelation was performed using SuperSignal West Dura Extended Duration Substrate solution (34075, Thermo Scientific) for $5 \mathrm{~min}$ in the dark and imaged with a Biorad Chemidoc. Images were treated with Image Lab v5.1. 


\section{Oligopaint probe design and preparation}

515 Oligopaint libraries were constructed following the procedures described in ${ }^{22}$; see the Oligopaints 516 website (https://oligopaints.hms.harvard.edu) for further details. Libraries were ordered from 517 CustomArray (Bothell, WA) in the 92K Oligo pool format. Coordinates (mm10), size, number, 518 density of probes and primers used for libraries for libraries are listed in Extended Data 9. Oligopaint 519 libraries were discovered using the archive $\mathrm{mm} 9$ bed files or the mm10 "balance" bed files ${ }^{22,23,44}$, 520 which consist of 32 mer or 35-41-mer genomic sequences throughout the regions of interest, 521 respectively. Bed files can be retrieved from the Oligopaints website 522 (https://oligopaints.hms.harvard.edu). Each library contains a universal primer pair followed by a 523 specific primer pair hooked to the genomic sequences (114-116 or 117-125 mers in total, 524 respectively). Oligopaint libraries were produced by emulsion PCR amplification from 525 oligonucleotide pools followed by a "two-step PCR" procedure and the lambda exonuclease method 526 described in ${ }^{22}$. The "two-step PCR" leads to secondary oligonucleotide-binding sites for signal 527 amplification with a secondary oligonucleotide (Sec1 or Sec6) containing two additional fluorophores, 528 each oligonucleotide carrying three fluorophores in total. ATTO-565 and Alexa-488 or ATTO-647 fluorophores were used for 2-color imaging. All oligonucleotides used for Oligopaint production were purchased from Integrated DNA Technologies (IDT, Leuven, Belgium). The Drosophila Oligopaint probe corresponds to the TAD2 probe on $\mathrm{X}$ chromosome described in ${ }^{20}$. Oligonucleotide primer sequences $\left(5^{\prime}->\right.$ 3') used in this study are listed in Supplementary Table 1.

FISH

The FISH protocol is described in ${ }^{45}$. Briefly, cells were grown directly on coverslips $[170 \pm 5 \mu \mathrm{m}$ (Zeiss)] and washed once with PBS. For cell-cycle analysis, cells were resuspended at a concentration of $1.5 \times 10^{6}$ cells/mL and deposited on coverslips coated with poly-L-lysin (P8920, Sigma-Aldrich; $0.01 \% \mathrm{w} / \mathrm{v}$ in water) for 10 minutes in a humid chamber. For Drosophila, four to five dechorionated and selected male embryos were squeezed directly on poly-L-lysin coated coverslips with a Dumont \#55 tweezer prior fixation (see more details in ${ }^{20}$ ). Cells were fixed for $10 \mathrm{~min}$ in $\mathrm{PBS} / 4 \%$ paraformaldehyde, rinsed in PBS, permeabilized for $10 \mathrm{~min}$ in $\mathrm{PBS} / 0.5 \%$ Triton $\mathrm{X}-100$, rinsed in PBS, incubated for $10 \mathrm{~min}$ in $0.1 \mathrm{M} \mathrm{HCl}$ and rinsed in $2 \times \mathrm{SSC} / 0.1 \%$ Tween $20(2 \times \mathrm{SSCT})$. Cells were then incubated for $20 \mathrm{~min}$ in $50 \%$ formamide/ $2 \times \mathrm{SSCT}$ at room temperature and for $20 \mathrm{~min} 50 \%$ formamide $/ 2 \times \mathrm{SSCT}$ at $60^{\circ} \mathrm{C}$. Probe mixtures contained Oligopaint probe at $\sim 1-3 \mu \mathrm{M}$ final concentration with the same amount of their secondary oligo and $0.8 \mu \mathrm{L}$ of RNase A in $20 \mu \mathrm{L}$ of FISH Hybridization Buffer [FHB; $50 \%$ formamide, $10 \%$ dextran sulfate, $2 \times \mathrm{SSC}$, and salmon sperm DNA 
$547(0.5 \mathrm{mg} / \mathrm{ml})]$. Probe mixtures were added directly to coverslips that were then sealed on glass slides

548 with rubber cement (Fixogum, Marabu). Cell DNA was denaturated for 3 min at $80^{\circ} \mathrm{C}$ on a heating 549 block immersed in a water bath, and probes were hybridized overnight at $42^{\circ} \mathrm{C}$ in a dark and humid 550 chamber. Cells were then washed for $15 \mathrm{~min}$ in $2 \times \mathrm{SSCT}$ at $60^{\circ} \mathrm{C}, 10 \mathrm{~min}$ in $2 \times \mathrm{SSCT}$ at room 551 temperature, $10 \mathrm{~min}$ in $0.2 \times \mathrm{SSC}$, and twice in PBS. For FISH in NPCs, the FISH protocol was 552 followed by an immunostaining after these washing steps (see Immunostaining procedure). Cells were 553 then incubated $10 \mathrm{~min}$ in PBS with DAPI (final concentration at $1 \mu \mathrm{g} / \mathrm{mL}$ ) and washed again at least 554 three times for 5 min each in PBS. For DAPI analysis without FISH, cells were fixed, permeabilized, 555 washed in PBS and incubated with DAPI as described above. Coverslips were mounted on slides with 556 Vectashield (CliniSciences) or Abberior liquid mounting media for STED imaging and sealed with 557 nail polish.

558

\section{Immunostaining}

560 After the washes of the FISH procedure following probe hybridizations, cells were washed in PBS/0.1\% Tween 20 (PBT) and incubated for 1 hour in PBT/2\%BSA. Incubation with an anti-Pax6 rabbit polyclonal antibody (PRB-278P-0100, Covance; 1:1000 dilution in $\mathrm{PBT} / 2 \% \mathrm{BSA}$ ) was performed overnight at $4^{\circ} \mathrm{C}$ between coverlips and glass slides in a humid and dark chamber. Cells were then washed in PBT and incubated with a secondary antibody (Donkey anti-Rabbit Alexa-647, A31573, Invitrogen; 1:200 dilution in $\mathrm{PBT} / 2 \% \mathrm{BSA}$ ) for 1 hour at room temperature between coverlips and glass slides in a humid and dark chamber. Cells were then washed in PBT, incubated in PBS with DAPI (final concentration at $1 \mu \mathrm{g} / \mathrm{mL}$ ) and washed again at least three times for 5 min each in PBS. Coverslips were mounted on slides with Vectashield (CliniSciences) and sealed with nail polish.

\section{Image acquisition}

3D-SIM imaging was performed with a DeltaVision V4 microscope equipped with a $\times 100 / 1.4$ numerical aperture (NA) Plan Super Apochromat oil immersion objective (Olympus) and electronmultiplying charge-coupled device (EMCCD; Evolve 512B, Photometrics) cameras for a pixel size of $80 \mathrm{~nm}$. Diode lasers at 405, 488, 561 and $647 \mathrm{~nm}$ were used with the standard corresponding emission filters. $Z$-stacks were acquired by scanning the sample in the axial direction ( $z$-step of $125 \mathrm{~nm}$ ) using

577 five phases and three angles per image plane. Raw images were reconstructed using SoftWorx 578 (version 6.5, GE Healthcare) using channel-specific optical transfer functions (pixel size of 579 reconstructed images $=40 \mathrm{~nm}$ ). Quality of reconstructed images was assessed using the SIMcheck 
580

581

582

583

584

585

586

587

588

589

590

591

592

593

594

595

596

597

598

599

600

601

602

603

604

605

606

607

608

609

610

611

612

plugin of Image ${ }^{46}$. Conventional wide-field images were generated from raw images by averaging angles and phases for each plane.

STED imaging was performed with an Expert line (Aberrior instruments) using an X83 inversed microscope equipped with a $\times 100 / 1.4$ NA oil immersion objective (Olympus). FISH probes labelled with ATTO-647 were observed by using a $640 \mathrm{~nm}$ excitation laser line at $40 \%$ of power combined with a depletion completed with a $775 \mathrm{~nm}$ laser at $30 \%$ of power. Images were collected with a pixel size of $20 \mathrm{~nm}$ and a dwell time of $10 \mu$ s with an averaging of 2 .

\section{Image analysis}

For 2-color FISH, raw super-resolution reconstructed channels were aligned using Chromagnon software ${ }^{47}$ using as reference files images of probes simultaneously labeled with the two corresponding fluorophores. Super-resolution reconstructed images were processed using the "Threshold and 16-bit conversion" utility of the SIMcheck plugin of ImageJ ${ }^{46}$ for further analysis. Image analysis was conducted using MATLAB (R2018-2019) and its “image processing toolbox". Region of interests (ROIs) of $2.2 \times 2.2 \mu \mathrm{m}$ throughout all $z$-slices surrounding FISH loci were extracted for further automated processing. Images were smoothed using 3D Gaussian filters $(\sigma=$ 0.5 ), and FISH probes were segmented in 3D using Otsu's method. Segmented objects smaller than $0.04 \mu \mathrm{m}^{3}\left(0.008 \mu \mathrm{m}^{3}\right.$ for the small $5 \mathrm{~d} 2$ probe and for Drosophila TAD) or in contact to the image border were discarded. Images with more than one segmented object per channel were discarded (except for the analysis of unpaired sister chromatids in RAD21-depleted cells, in which only images with two segmented objects were kept for the analysis). Segmented images were systematically verified by visual inspection. All quantitative analysis of volumes, densities, sphericities, principal axis lengths and CND numbers and sizes were performed using probes labeled with ATTO-565 fluorophore to avoid eventual differences coming from resolution (out of the 26 designed probes, \#51a and \#102a were not analyzed in Extended Data 8b, c, e and f). Volumes were calculated by multiplying the number of segmented voxels by the voxel volume. Densities were calculated by dividing the genomic size (in $b p$ ) of the probes by their mean volume. Principal axis lengths were defined as the lengths of the major axes of ellipsoids that have the same normalized second central moments as the segmented probes. Sphericity scores $\psi$ were calculated using the following formula:

$\psi=\frac{\pi^{\frac{1}{3}}(6 V)^{\frac{2}{3}}}{A}$ in which $V$ is a volume of the segmented object and $A$ is its surface area (a sphericity of 1 corresponds to a perfect sphere). OFs were calculated using Jaccard Index of the segmented probes labeled in different colors and 3D distances were calculated between their centroids. PCCs were calculated using the intensities (scaled from 0 to 1 ) of the two channels from maximum projected 
images. To generate averaged images, individual segmented probes of each channel were first projected along the axial direction and the 2D distance between their centroid was calculated. For each color, all the binary segmented images were centered using probe centroids and summed. Composite images were generated by shifting the two different channels along one axis using the mean $2 \mathrm{D}$ distance calculated between the centroids. For CND quantification, the watershed function (image processing toolbox) was applied to the complement of the scaled intensity values ( 0 to 1 , without Gaussian filter) within ROIs defined by the full probe segmentations described above. Watershed segmented objects smaller than $0.0072 \mu \mathrm{m}^{3}$ were discarded. An independent CND quantification was performed using ImageJ software: images were maximum projected along the $z$-axis before segmentation using Otsu's method, and local intensity maxima above Otsu's threshold value within the largest segmented object were identified using the "Find Maxima" utility (noise tolerance $=10$ ). For DAPI analysis, a single $z$-slice was extracted and DAPI was segmented using Otsu's method; objects smaller than $0.0144 \mu \mathrm{m}^{2}$ were discarded. Watershed was then applied as described above within the ROI defined by DAPI segmentation. For comparison with FISH, a single $z$-slice was randomly selected between the minimum and maximum $z$ coordinate of FISH segmented object. Watershed was then applied as for DAPI, within ROIs defined by probe segmentation. For STED images, probes were first segmented using Otsu's method (images filtered using a Gaussian filter, $\sigma=$ 2; segmented objects smaller than $0.04 \mu \mathrm{m}^{2}$ were discarded), and watershed was applied as described above (objects smaller than $0.0036 \mu \mathrm{m}^{2}$ were discarded). Extrapolated diameters $d$ were calculated using the following formula $d=\sqrt{\frac{4 \times A}{\pi}}$ in which $A$ is the surface of the watershed segmented object. For cell-cycle analysis, conventional wide-field images were projected along the $z$-axis using average intensity and smoothed with a Gaussian filter $(\sigma=8)$ before DAPI segmentation using Otsu's method. Segmented nuclei touching image borders or smaller than $38.4 \mu \mathrm{m}^{2}$ were discarded and individual nuclei contacting each other were further separated using watershed segmentation within the ROIs defined by DAPI segmented objects. FISH was then analyzed within nuclei classified as G1 based on both their areas and their intensities.

\section{Hi-C analysis}

Publicly available ESC, NPC and ncxNPC Hi-C data ${ }^{27}$ were downloaded from GEO database (GSE96107). Raw Hi-C interaction counts were computed using in-house R scripts using the "misha" package (https://github.com/msauria/misha-package). Hi-C enrichment scores were computed using raw mapped interactions. Juicer $\mathrm{KN}$ normalized data ${ }^{48}$ were used for map visualization $(5 \mathrm{~kb}$ resolution) and for correlations with probe densities. Publicly available serum diploid ESC scHi-C maps were downloaded from GEO database (GSE94489). More specifically, mm9 aligned Hi-C 
652

653

654

655

656

657

658

659

660

661

662

663

664

665

666

667

668

669

670

671

672

673

674

675

\section{1}

74

676 Data availability statement

misha tracks obtained from GEO were lifted to the mm10 mouse genome reference using the 'gtrack.liftover' function of the misha R package. To account for high sparsity of scHi-C maps we divided all available maps (821 maps with coverage higher than 100,000 pairs) into 5 increasing coverage quantiles. Finally, only the top 20\% maps (165 maps with coverage between 855,974 and 2,463,598 pairs) were included in subsequent analyses.

\section{Collection and alignment of ESC and NPC ChIPseq, ATACseq and RNAseq datasets}

Publicly available ESC and NPC ChIP-Seq data for H3K4me1, H3K4me3, H3K27Ac and H3K27me3 marks, ATAC-Seq and RNA-Seq data used in this study are listed in Supplementary Table $2^{27,49,50}$. Raw ChIP-Seq and ATAC-Seq sequencing data were collected and aligned to the mm10 version of the mouse genome using bowtie 2 with default parameters [v2.1.0, $\left.{ }^{51}\right]$. Reads with low mapping quality $(\mathrm{mapq}<30)$ were discarded using samtools (v1.10) ${ }^{52}$. RPKM-normalized bigwig binary files were generated using the bamCoverage function from Deeptools2 (v2.5.1) ${ }^{53}$ with the following parameters: -of=bigwig --samFlagExclude 128 --ignoreDuplicates -e 200 -normalizeUsingRPKM. Finally, replicates were merged using the bigWigMerge and bedGraphToBigWig tools from UCSC ${ }^{54}$ with default parameters. Raw RNA-Seq data were aligned to the mm10 reference genome using STAR version 2.5.0b. PCR duplicates were removed, and RPM normalized coverage tracks were produced using STAR in "inputAlignmentsFromBAM" runMode. All the produced BigWig files were imported into $\mathrm{R}$ using the misha framework and probe signal quantification was computed as global percentile.

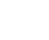

8

9

0

2

\section{os}


677

678

679

680

681

682

683

684

685

686

687

688

689

690

691

692

693

694

695

696

697

698

699

700

701

702

703

704

705

706

707

708

709

710

711

712

713

714

715

716

717

718

719

The datasets generated during and/or analyzed during the current study are available from the corresponding authors on request. Publicly available datasets used in this study (GSE84646, ENCSR000CGO， ENCSR032JUI， ENCSR000CGQ， ENCSR059MBO， ENCSR253QPK, ENCSR857MYS, GSE96107, GSE74330) are detailed in Supplementary Table 2.

\section{Code availability}

The codes used in the current study are available on request.

\section{Methods-only references}

42 Saldana-Meyer, R. et al. RNA Interactions Are Essential for CTCF-Mediated Genome Organization. Mol Cell 76, 412-422 e415, doi:10.1016/j.molcel.2019.08.015 (2019).

43 Gaspard, N. et al. Generation of cortical neurons from mouse embryonic stem cells. Nature protocols 4, 1454-1463, doi:10.1038/nprot.2009.157 (2009).

44 Beliveau, B. J. et al. Versatile design and synthesis platform for visualizing genomes with Oligopaint FISH probes. Proc Natl Acad Sci U S A 109, 21301-21306, doi:10.1073/pnas.1213818110 (2012).

45 Szabo, Q., Cavalli, G. \& Bantignies, F. Higher-Order Chromatin Organization Using 3D DNA Fluorescent In Situ Hybridization. Methods Mol Biol 2157, 221-237, doi:10.1007/978-1-0716-0664-3_13 (2021).

46 Ball, G. et al. SIMcheck: a Toolbox for Successful Super-resolution Structured Illumination Microscopy. Sci Rep 5, 15915, doi:10.1038/srep15915 (2015).

47 Matsuda, A., Schermelleh, L., Hirano, Y., Haraguchi, T. \& Hiraoka, Y. Accurate and fiducial-marker-free correction for three-dimensional chromatic shift in biological fluorescence microscopy. Sci Rep 8, 7583, doi:10.1038/s41598-018-25922-7 (2018).

48 Durand, N. C. et al. Juicer Provides a One-Click System for Analyzing Loop-Resolution Hi-C Experiments. Cell systems 3, 95-98, doi:10.1016/j.cels.2016.07.002 (2016).

49 Kloet, S. L. et al. The dynamic interactome and genomic targets of Polycomb complexes during stemcell differentiation. Nat Struct Mol Biol, doi:10.1038/nsmb.3248 (2016).

$50 \mathrm{Xu}$, J. et al. Landscape of monoallelic DNA accessibility in mouse embryonic stem cells and neural progenitor cells. Nat Genet 49, 377-386, doi:10.1038/ng.3769 (2017).

51 Langmead, B. \& Salzberg, S. L. Fast gapped-read alignment with Bowtie 2. Nat Methods 9, 357-359, doi:10.1038/nmeth.1923 (2012).

$52 \mathrm{Li}, \mathrm{H}$. et al. The Sequence Alignment/Map format and SAMtools. Bioinformatics 25, 2078-2079, doi:10.1093/bioinformatics/btp352 (2009).

53 Ramirez, F. et al. deepTools2: a next generation web server for deep-sequencing data analysis. Nucleic Acids Res 44, W160-165, doi:10.1093/nar/gkw257 (2016).

54 Kent, W. J., Zweig, A. S., Barber, G., Hinrichs, A. S. \& Karolchik, D. BigWig and BigBed: enabling browsing of large distributed datasets. Bioinformatics 26, 2204-2207, doi:10.1093/bioinformatics/btq351 (2010).

55 Roukos, V., Pegoraro, G., Voss, T. C. \& Misteli, T. Cell cycle staging of individual cells by fluorescence microscopy. Nat Protoc 10, 334-348, doi:10.1038/nprot.2015.016 (2015). 
a

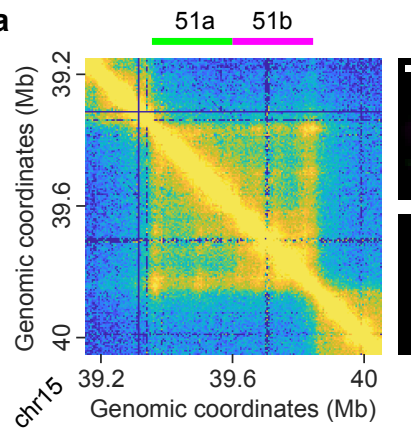

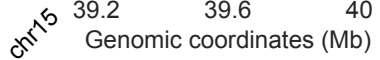
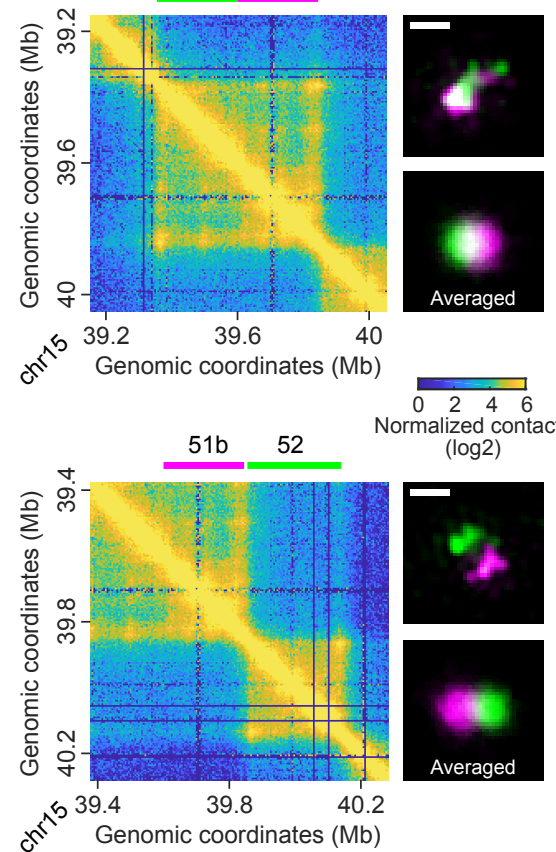

b

:

8

㩆

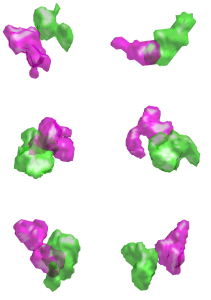

C
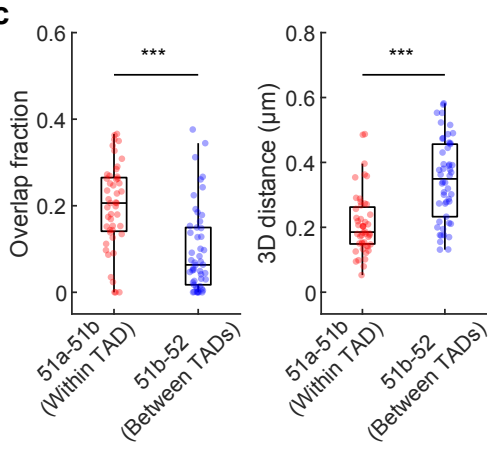

d 15 pairs of probes:
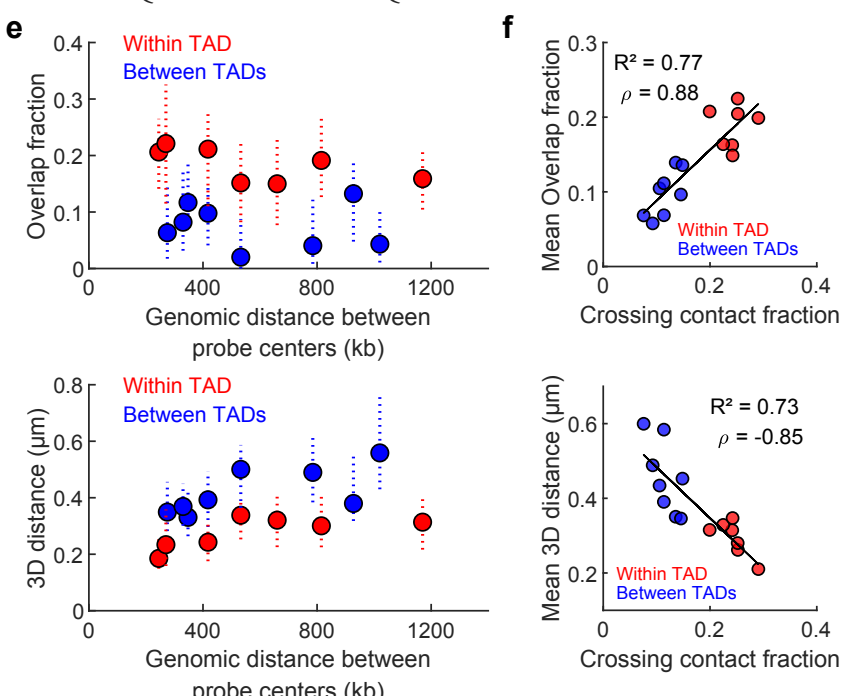
a

b

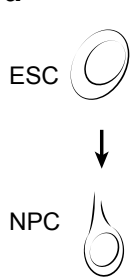

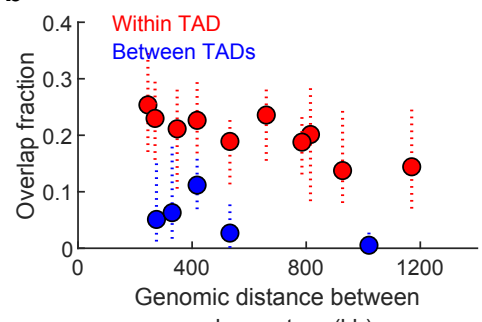

probe centers $(\mathrm{kb})$

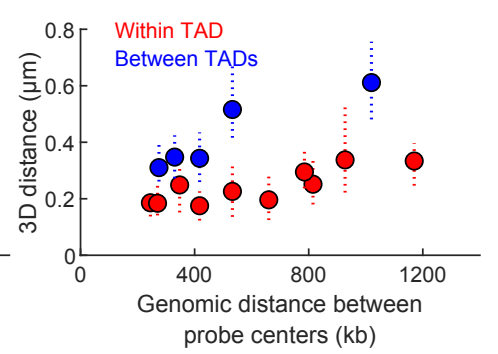

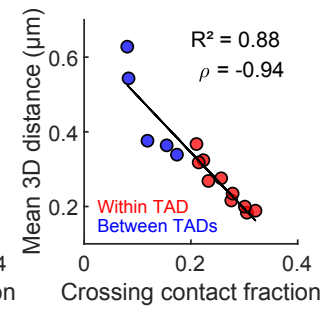

Crossing contact fraction

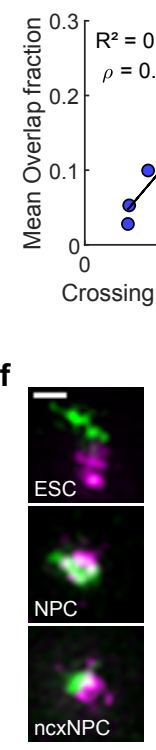

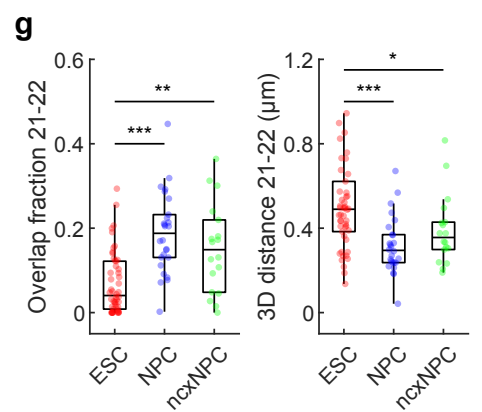
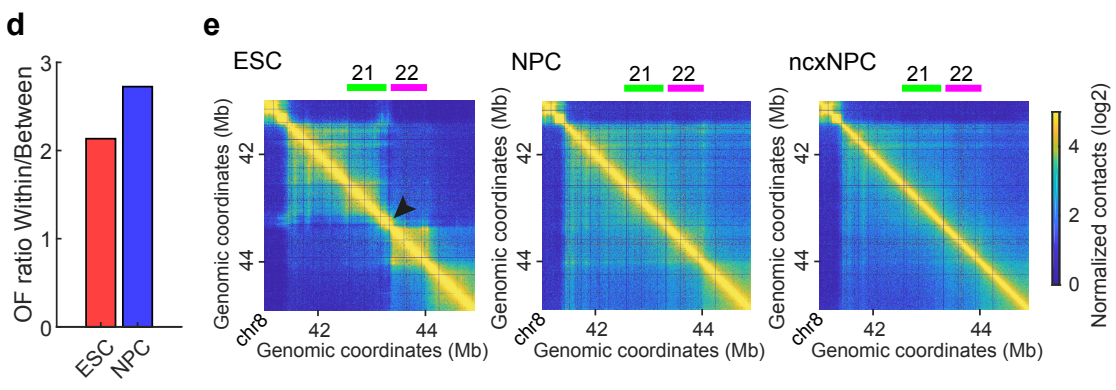


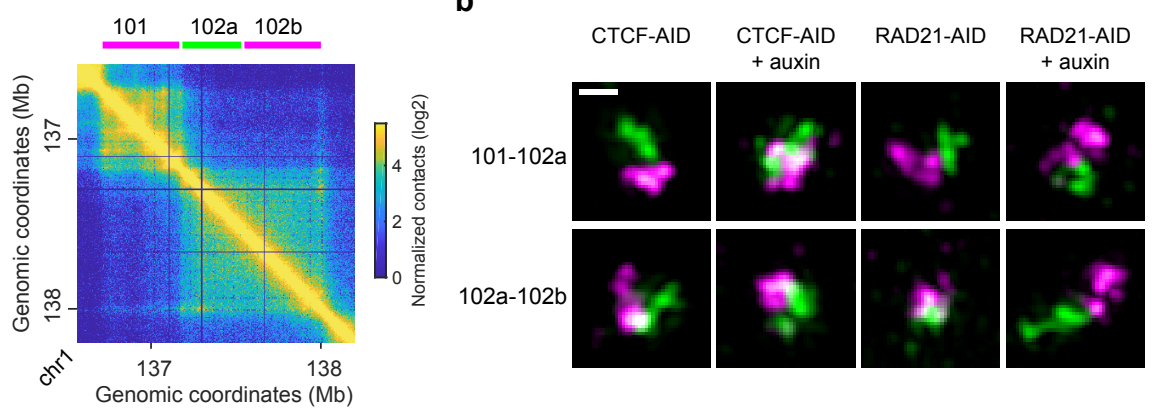

C

CTCF-AID

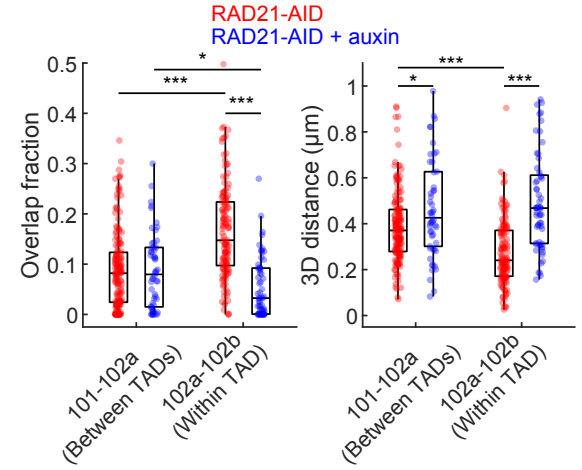

d
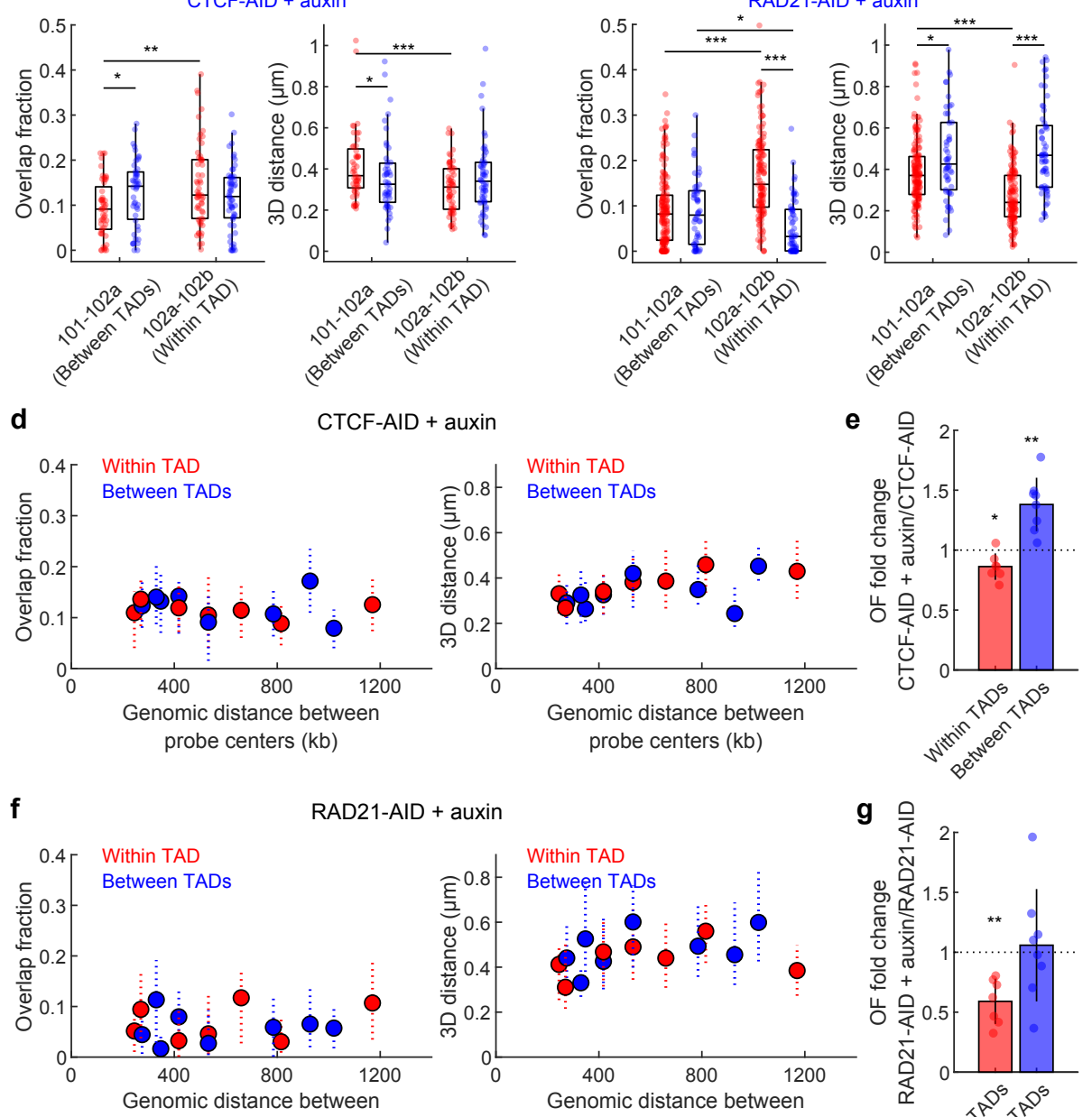

probe centers $(\mathrm{kb})$

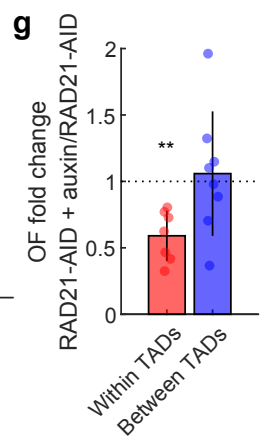



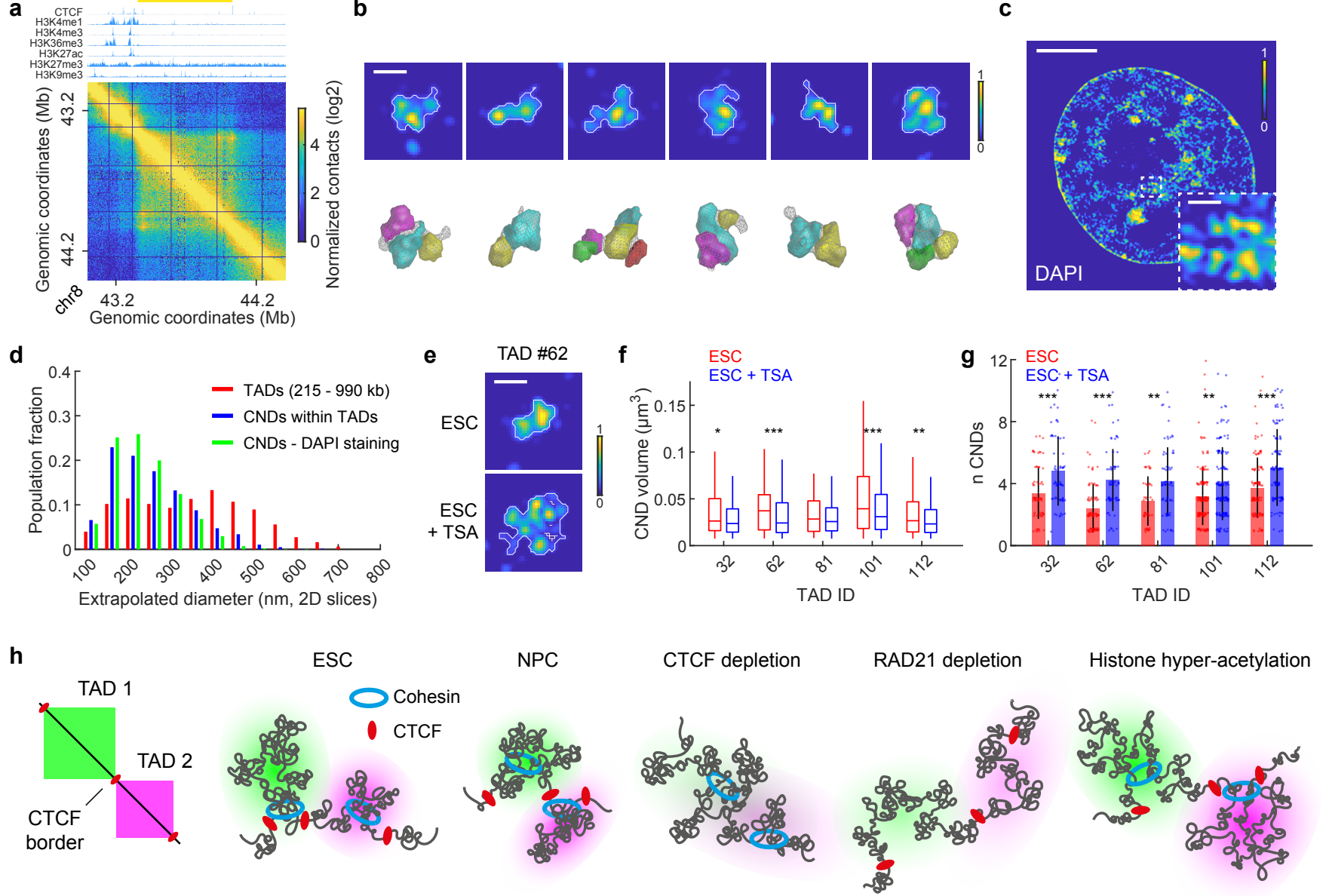

Histone hyper-acetylation

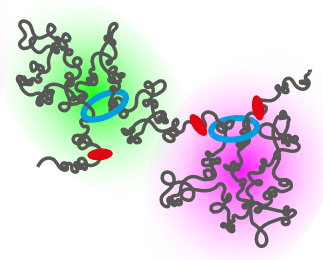

